\title{
Artículos
}

\section{Identidades masculinas rarámuris ante la migración y la sobrevivencia}

\section{Rarámuri male identities in the face of migration and survival}

\author{
Beatriz Martínez Corona* \\ José Álvaro Hernández Flores**
}

\begin{abstract}
Resumen
La vulnerabilidad y la pobreza que prevalecen en la Sierra Tarahumara, en Chihuahua, México, obligan a sus habitantes a migrar en búsqueda de mejores condiciones de vida. Esta migración tiene repercusiones en las identidades y relaciones de género. Con base en una amplia revisión bibliográfica y en el trabajo de campo desarrollado con jornaleros migrantes rarámuris, se analizan las transformaciones identitarias y genéricas experimentadas históricamente por varones pertenecientes a este grupo étnico. Destacan, en particular, los cambios derivados de su participación como fuerza de trabajo en los circuitos migratorios asociados a la agroindustria, y los procesos de adaptación o resistencia frente al régimen de acumulación flexible que los explota, excluye y margina.
\end{abstract}

Palabras clave: masculinidades; etnia; género; migración; rarámuris.

\begin{abstract}
The vulnerability and poverty that prevail in the Sierra Tarahumara, in Chihuahua, Mexico force its inhabitants to migrate in search of better living conditions. This migration has repercussions on gender identities and relations. On the basis of an extensive bibliographical review and fieldwork undertaken with migrant Rarámuri laborers, the identity and gender transformations historically experienced by males belonging to this ethnic group are analyzed. Particular attention is paid to the
\end{abstract}

* Colegio de Postgraduados, campus Puebla. Dirección postal: Km 125.5 carretera federal México-Puebla, 72760, Santiago Momoxpan, Puebla, México.Correo electrónico: beatrizm@ colpos.mx ORCID: http://orcid.org/0000-0002-0745-4270

** El Colegio de México, A.C., Centro de Estudios Demográficos, Urbanos y Ambientales. Dirección postal: Carretera Picacho-Ajusco 20, Tlalpan, 14110, Ciudad de México, México. Correo electrónico: jalvaro@colmex.mx ORCID: https://orcid.org/0000-0002-8349-0505

D.R. (C) 2019. Estudios Demográficos y Urbanos Licencia Creative Commons Atribución-NoComercial-SinDerivar (CC BY-NC-ND) 4.0 Internacional 
changes derived from their participation as a work force in migration circuits associated with agro-industry, and the processes of adaptation or resistance to the flexible accumulation regime that exploits, excludes and marginalizes them.

Keywords: masculinities; ethnicity; gender; migration; Rarámuris.

\section{Introducción}

La masculinidad suele definirse como el conjunto de atributos, valores, funciones y conductas que se suponen esenciales al varón en una cultura determinada. Los estudios de género refieren la existencia de un modelo hegemónico de masculinidad (Connel y Messerschimdt, 2005), el cual constituye un esquema cultural socialmente construido, en el que se presenta al varón en una posición de ventaja y dominio con respecto a las mujeres. Es a partir de este modelo que se construyen las identidades masculinas, se discrimina y subordina a las mujeres, así como a otros hombres que no se adaptan a él (De Keijzer, 1997).

La perspectiva de género, como señala Díaz (2014, p. 361), facilita comprender "el carácter relacional, diverso y complejo de las y los sujetos indígenas, como resultado de un proceso constante y conflictivo de la socialización de sistemas simbólicos occidentales y las resistencias colectivas de la identidad indígena". La construcción de la masculinidad ocurre en el marco de una estructura ideológica desde donde se decide, emite y modela esa conducta. En ese sentido, se considera que los hombres construyen sus masculinidades a partir de dos ejes: uno que atraviesa las vivencias individuales y cotidianas; $y$ otro en el que interviene la sociedad, expresada en sus instituciones fundamentales (la familia, la religión, el Estado, el mercado) (De Keijzer, 1997).

A partir de información histórica, documental, etnográfica, así como de entrevistas a profundidad y grupos focales realizados en los campos agrícolas de Cuauhtémoc, Chihuahua, durante 2010 y 2011, el presente trabajo se plantea como objetivo profundizar en los cambios y resignificación de las masculinidades de los jornaleros agrícolas rarámuris, a partir de su incorporación a los circuitos migratorios regionales y de la venta de su fuerza de trabajo al sector agroindustrial.

Se parte de la hipótesis de que las descripciones del sistema sexo/género (Rubin, 1986) prevalecientes en las comunidades rarámuris, reportadas en las fuentes etnográficas y etnohistóricas clásicas -las cuales enfatizan la flexibilidad de las relaciones afectivo-sexuales y las condiciones de relativa igualdad que priman en los espacios domésticos-, no implican una modifi- 
cación en el patrón genérico basado en la dominación masculina. Partiendo de esta premisa, se considera que la integración de la etnia rarámuri a los circuitos migratorios de tipo pendular, vinculados a la agroindustria, pone en marcha procesos de intercambio sociocultural que derivan en un replanteamiento de las nociones tradicionales de género, entre ellas las que se refieren de manera específica a las masculinidades.

En los siguientes apartados se describen, desde una perspectiva etnohistórica, los rasgos principales del sistema sexo/género de la población rarámuri. Posteriormente se identifican, a partir de una perspectiva diacrónica, los acontecimientos históricos que significaron cambios en las construcciones sociales de género, en particular aquellos que se vinculan con la integración de la etnia rarámuri a los circuitos migratorios regionales, así como las posibilidades y limitaciones que ofrece este nuevo contexto para el establecimiento de relaciones de género menos inequitativas.

\section{La etnia rarámuri}

El pueblo rarámuri pertenece, junto con los pueblos pimas, guarojíos y tepehuanos, entre otros, a los denominados "grupos indígenas sonorenses" que habitan actualmente en el estado de Chihuahua, al noroeste de México, en la Sierra Tarahumara. De acuerdo con el INEGI (2010), de las más de 109000 personas que hablan alguna lengua indígena en dicha entidad, poco más de 85000 pertenecen al pueblo rarámuri, el cual constituye el grupo étnico mayoritario en esta región.

Antes de la conquista española, el pueblo rarámuri se asentaba en los valles ubicados en las estribaciones orientales de la Sierra Madre Occidental y en las llanuras contiguas a este macizo montañoso (Kennedy, 1970). No obstante, tras siglos de persecuciones, escaramuzas y repliegues sucesivos, la población rarámuri asumió una estrategia de resistencia pasiva frente a los invasores, la cual supuso su huida hacia las regiones más recónditas e inaccesibles de la Sierra Tarahumara (Neumann y González, 1991). El aislamiento geográfico les permitió estabilizarse demográficamente e incluso aumentar su población, aunque a un ritmo menor que el de la población mestiza, y al costo de una pobreza económica vinculada, sobre todo, a la baja productividad de sus tierras, misma que en los últimos años se ha visto afectada por fenómenos como la deforestación, la erosión y los cambios ambientales (Rodríguez, 1999).

Actualmente, la población rarámuri se encuentra dispersa a lo largo de la Sierra Tarahumara, la cual forma parte de la Sierra Madre Occidental, 
localizada en el estado de Chihuahua, al noroeste de México. El territorio de la Sierra Tarahumara se extiende a lo largo de 60000 kilómetros cuadrados, en un terreno accidentado cuya altitud oscila entre los 300 y 3000 metros sobre el nivel del mar. La principal característica de los grupos étnicos que habitan este territorio es que se encuentran ubicados en asentamientos dispersos. De acuerdo con datos del Gobierno del Estado de Chihuahua (2006), de las casi siete mil comunidades que hay en la Sierra Tarahumara, $86 \%$ poseen menos de 50 habitantes y se encuentran ubicadas en lo más recóndito de la geografía serrana. Asimismo, se señala que más de la mitad de la población rarámuri se concentra en rancherías donde habitan menos de cien personas.

La dispersión poblacional, aunada a las dificultades para acceder a dichos asentamientos, ha limitado la infraestructura y el acceso a los servicios por parte de este grupo étnico: $90.6 \%$ no cuenta con servicios de salud, $78.9 \%$ carece de agua entubada, $96.6 \%$ no tiene drenaje, $91.6 \%$ no posee energía eléctrica y $80.5 \%$ de las viviendas tienen piso de tierra. Por otro lado, el acceso a la educación formal, escolarizada, es muy deficiente. Datos sobre la escolaridad de los rarámuris indican que $43 \%$ son analfabetos, $52.7 \%$ tienen algún grado de educación básica y $14.2 \%$ son monolingües (INALI, 2010).

La población rarámuri vive principalmente de la agricultura. No obstante, dadas las condiciones agroecológicas que priman en este territorio ( $95 \%$ de la tierra agrícola es de temporal, con suelos pobres y delgados que resultan poco aptos para la agricultura), dicha actividad apenas alcanza para cubrir sus necesidades alimenticias, y en ocasiones, ni eso. A esta precaria situación habría que sumar la incidencia de fenómenos climáticos (heladas y sequías), que en las últimas décadas se han vuelto crónicos y han propiciado episodios de hambruna en los municipios serranos (Acuña, 2007).

El acceso limitado a los alimentos se traduce en altos niveles de desnutrición que afectan sobre todo a los niños y que se reflejan en la tasa de mortalidad infantil más grande de todo México (23\%). Esta condición no sólo afecta a la población infantil, sino también a las mujeres, sobre todo a las embarazadas y a las que están en periodo de lactancia. Datos del Gobierno del Estado de Chihuahua (2006) señalan que $40 \%$ de las mujeres tarahumaras tienen anemia. Esta situación, aunada a la carencia de servicios básicos de salud, ha propiciado que las infecciones gastrointestinales, la influenza y la neumonía -enfermedades que con un adecuado tratamiento no son mortales- sean la principal causa de muerte entre los indígenas tarahumaras (Gabrielova, 2007). De esta manera, los niveles de vida en la Sierra Tarahumara son dos veces inferiores al promedio nacional, y en el caso de los 
municipios que albergan población rarámuri, tres veces inferiores a dicho promedio (Stefani y Arteaga, 1995). El Programa de Naciones Unidas para el Desarrollo informa, por ejemplo, que el municipio de Batopilas, Chihuahua, presentó un Índice de Desarrollo Humano (IDH) de su población indígena de " 0.3010 , cifra menor que el país con menor desarrollo humano en el mundo, que es Níger, con un IDH de 0.3300" (PNUD, 2010, p. 31).

Las condiciones de pobreza que prevalecen en las localidades de origen de la población rarámuri migrante, aunadas a la intensificación de la violencia vinculada al crimen organizado y a las limitantes que enfrentan las familias rarámuris para abastecer su propio consumo, han propiciado procesos migratorios de carácter definitivo hacia las zonas urbanas de Chihuahua, Ciudad Juárez y Creel; y de tipo temporal a los campos agrícolas de Sonora, Baja California, Sinaloa, Durango y Chihuahua.

El proceso de integración de la mano de obra indígena a los mercados agrícolas, como fuerza de trabajo flexible, segmentada por etnia, género y edad, ha sido ampliamente documentado en México (Lara, 1998; Lara y Carton de Grammont, 2011; Cárdenas, 2014). Para el caso rarámuri, sin embargo, el fenómeno reviste ciertas particularidades, entre las que destaca el establecimiento de relaciones laborales y de convivencia con otros grupos sociales como los menonitas, los mestizos o "chabochis", y otros grupos étnicos con los que comparten el espacio migratorio (Martínez y Hernández, 2011).

El contacto con otros grupos o personas, en contextos culturales distintos al propio, conlleva inevitablemente procesos de intercambio y reconstitución identitaria durante los cuales algunos sujetos mantienen intactos los rasgos de su cultura, en tanto que otros experimentan múltiples transformaciones y adaptaciones (Raesfeld, 2006). Los encuentros y las confrontaciones interétnicas que se dan entre la población migrante y la sociedad receptora suelen trastocar el sentido de etnicidad de los primeros, actuando sobre la subjetividad colectiva de los grupos culturalmente identificados con sus raíces ancestrales, las cuales son actualizadas a través de procesos complejos de resignificación, donde los contenidos de género están relacionados con los contextos históricos (Díaz, 2011). A partir de este contexto, el presente trabajo pretende documentar transformaciones en las relaciones de género y en las masculinidades de los jornaleros rarámuris involucrados en este proceso. 


\section{El sistema sexo-género rarámuri}

Las características culturales del grupo étnico rarámuri han sido documentadas por diversos investigadores que dan cuenta de sus particularidades (Rodríguez, 1999; Pintado, 2000; Pérez, 2003). La literatura académica, sin embargo, ha explorado poco la construcción cultural de género en el interior de este grupo étnico, y es aún más escasa la información sobre la forma específica en que se construyen y expresan las identidades masculinas, y las características de las relaciones de género que establecen.

Entre los pueblos indígenas, los mitos y leyendas suelen explicar algunas de las principales diferencias socioculturales que existen entre hombres y mujeres. El mito rarámuri sobre el origen, sin embargo, no hace alusión alguna a diferencias u oposiciones destacadas con relación al género. Si acaso, el mito de origen rarámuri refiere a una oposición de tipo étnico-racial: Onorúame (el dios que es padre y madre a la vez) coció dos figuras humanas, a la primera la dotó de alma con un soplo para crear al rarámuri, en tanto que a la segunda la coció de forma inadecuada, dejándola blanda y blanca, para crear al chabochi (hombre blanco y barbudo) (Acuña, 2007). Este relato corresponde a la tendencia de la investigación antropológica sobre la "otredad" que, en este caso, plantea que la construcción identitaria de la persona rarámuri se construye de acuerdo con un sistema de categorías o atributos que se desarrollan en oposición al no rarámuri o chabochi (mestizo o criollo).

No obstante, en la cosmovisión de este grupo étnico predominan algunas nociones de lo masculino y lo femenino que conviene hacer notar. El Sol, por ejemplo, se concibe como la entidad protectora de los varones; en tanto que la Luna es la de las mujeres, estableciendo con ello una dualidad por asociación de ideas con astros de características opuestas y complementarias (Acuña, 2007). Otra diferencia que vale la pena señalar concierne al número de almas que los rarámuris atribuyen a hombres y mujeres. Para los rarámuris, la vida deviene del alma o soplo divino que Onorúame imprimió a las figuras de barro primigenias. Al hombre le otorgó tres soplos y a la mujer cuatro. Si bien no existe consenso respecto a las razones que explican esta diferencia, la mayoría de los investigadores concuerdan en que el cuarto soplo se atribuye a las mujeres porque éstas son consideradas por los rarámuris como seres más fuertes y resistentes, sobre todo porque cuentan con la capacidad de engendrar un nuevo ser (Pintado, 2000; Acuña, 2007). Si bien el número de almas no está vinculado a diferencias de género -ya que para los rarámuris la debilidad o fortaleza de las almas depende de las relaciones de armonía con la divinidad y no de su condición genérica o biológica-, es posible apreciar la desigualdad en las relaciones de género a partir 
de la forma en que se distribuye el trabajo, así como la valoración social y simbólica que se atribuye a las tareas asignadas a las mujeres, la cual reproduce su posición subordinada.

Acuña (2007) reconoce las nociones que determinan los roles de género dentro de la etnia rarámuri mediante la identificación de las actividades asignadas a mujeres y hombres a lo largo de su ciclo vital. A partir de esta revisión es posible delinear algunas características del sistema sexo-género rarámuri, es decir, el conjunto de arreglos a partir de los cuales una sociedad transforma la sexualidad biológica en productos de la actividad humana (Rubin, 1986).

De acuerdo con Acuña (2007), la identidad rarámuri descansa sobre una serie de atributos, entre los que destacan: $a$ ) poseer entidad física (un cuerpo que posea los rasgos fenotípicos característicos del grupo étnico); b) entidad espiritual (almas); c) filiación consanguínea (en algún grado); y d) participación sociocultural (compartir los mismos esquemas de percepción y acción). La presencia de estos atributos permite afirmar una identidad étnica -la rarámuri-, pero además, sirve también como criterio para delimitar dos maneras distintas de ser rarámuri: como hombre y como mujer.

Los primeros años de vida de los rarámuris transcurren sin distinciones entre niños y niñas. Independientemente del sexo, al recién nacido se le llama muchí; sin embargo, a los pocos días de nacer serán llamados tewé si es niña o towi si es niño. Algunos autores señalan que es posible derivar el trato diferenciado que se le da a ambos sexos en la vida social a partir de la terminología empleada en el parentesco durante la infancia. Y es que mientras el padre usa un término diferente para designar al hijo (nó) y a la hija ( $m a ́)$, la madre emplea el mismo término para ambos (donalá). De igual modo, el niño y la niña utilizan términos diferentes para llamar al padre (onó/ mari), aunque ambos usan el mismo para llamar a la madre (naná) (Bennett y Zingg, 1978). A partir de este hecho, Acuña (2007) concluye que es muy posible que la madre cuide y trate a todos los hijos por igual, sin distinción de sexo, mientras que el padre no preste la misma atención a la hija, que está más en contacto con la madre, que al hijo, con el que pasa más tiempo.

Es hasta la segunda infancia, a partir de los diez u once años, cuando las actividades de niños y niñas comienzan a diferenciarse en función del sexo, realizando cada uno las tareas que les serán culturalmente más apropiadas después del matrimonio. Al respecto, es interesante apreciar cómo los juegos tradicionales reproducen tal diferenciación. La rowera (carrera con lanzamiento de aro) se reserva para las mujeres, mientras que el rarajípari (carrera con lanzamiento de bola) es para los hombres; pasa lo mismo con el nakibuti (lanzamiento de palitos unidos por un cordel) que practican las 
mujeres, en oposición a la ra'chuela (lanzamiento de bola) que sólo juegan los hombres. Llama la atención que los juegos de lanzamiento y puntería como el rujibara (cuatro con teja), el júbara (cuatro con palillos) y la choguira (lanzamiento con arco y flecha) son exclusivamente para varones, así como los juegos de azar (romayá) y también la lucha (najarápuami). ${ }^{1}$

Una vez que hombres y mujeres llegan a la pubertad, se considera que están listos para el matrimonio. Entre los rarámuris la iniciativa para formar una pareja recae por tradición en las mujeres, quienes atraen la atención de los varones en espera de ser correspondidas. Las parejas recién formadas pueden vivir juntas por espacio de algunos meses, periodo tras el cual pueden disolver su compromiso sin dificultades ni obligaciones (Velasco, 1987). Una vez que transcurre ese periodo de prueba, y que se ha obtenido el permiso de los progenitores y de las autoridades tradicionales, la pareja inicia su vida conyugal sin que medie ceremonia o fiesta especial alguna. El matrimonio conlleva un cambio de estatus con el cual se adquieren compromisos y responsabilidades que se consideran propios de las personas adultas. Una vez casados, los jóvenes rarámuris serán considerados como mukí, o esposa, y rejói, o marido. Aunque el matrimonio es monógamo y existe la exigencia tradicional de fidelidad, es posible que durante la vida marital se presenten rupturas y separaciones sin que eso implique culturalmente una sanción moral o social. No existe un patrón de residencia tradicional; éste puede ser matrilocal, patrilocal o neolocal, de acuerdo con las condiciones económicas y las circunstancias familiares de cada pareja; aunque dadas las condiciones de pobreza que priman en las localidades de la Sierra Tarahumara, es frecuente que las mujeres inicien su vida conyugal en la casa de sus suegros.

La mayoría de los investigadores coinciden en que las relaciones afectivas y sexuales están dotadas de cierto grado de flexibilidad (Gómez, 2009; Gabrielova, 2007; Acuña, 2007). El relajamiento del orden sexual suele ocurrir principalmente durante las fiestas, donde al calor del tesgüino se flexibilizan los criterios morales y se desafían las normas sociales. Acuña y Gómez (2012) señalan, por ejemplo, que no es raro presenciar durante las tesgüinadas, bromas y juegos de tipo homosexual entre los varones. Incluso

1 Es interesante apreciar que en algunos juegos la diferencia está dada por el tipo de objeto que se utiliza. En el caso de las carreras, por ejemplo, las mujeres persiguen un aro y los hombres una bola. Algunas interpretaciones asocian los atributos de los objetos con las características biológicas de los respectivos jugadores, y más aún, con los astros a los que se vinculan (por ejemplo: mujer-luna-aro en contraposición de hombre-sol-esfera). No obstante, algunos investigadores plantean que no existe evidencia dentro de la propia cultura que valide dichas conclusiones, por lo que en todo caso "sólo podemos decir que se trata de objetos complementarios, como complementarios son los papeles del hombre y la mujer para la producción y reproducción de la vida social" (Acuña, 2007, p. 51). 
es señalada la aceptación de los llamados nawikis, homosexuales que, de acuerdo con Gómez (2009, p. 695), "se definen no sólo por su preferencia sexual, sino que poseen interés por comportarse como el sexo opuesto". Asimismo, no son extraños los conflictos por infidelidades durante las fiestas. Se ha llegado a reportar, incluso, la existencia de algunos casos de poligamia (un hombre con varias esposas) y poliginia (una mujer con dos hombres) entre los rarámuris (Gómez, 2009). Aunque estas conductas no suelen ser bien vistas por la comunidad, tampoco se sancionan socialmente con severidad.

Aunque en el interior de las familias se mantiene la división tradicional del trabajo, es frecuente que hombres y mujeres sepan hacer todas las actividades que realiza su compañero. En el contexto de aislamiento propio de la serranía, el conocimiento y la práctica de ambos cónyuges con respecto a las actividades productivas y reproductivas funciona como medida preventiva para asegurar la supervivencia en caso de quedar solos por tiempo prolongado (Acuña y Gómez, 2012). Los hombres, por lo regular, se ocupan de las labores agrícolas, la caza, la recolección y acarreo de leña, la construcción y reparación de la vivienda. Las mujeres, por su parte, se ocupan del pastoreo y de las funciones reproductivas, como la crianza y el cuidado de los niños, el acarreo de agua, la elaboración de alimentos, la limpieza del hogar, el lavado de ropa, así como la elaboración de artesanías textiles y de cerámica.

El sistema testamentario entre los rarámuris es de tipo bilateral, lo que significa que hombres y mujeres reciben herencia por igual. De hecho, es práctica habitual que, aunque las tierras y los bienes se compartan durante el matrimonio, cada cónyuge mantiene el derecho de propiedad sobre lo suyo, derecho que seguirá acompañándoles ante una eventual separación. Esta medida garantiza la independencia económica de los cónyuges en el interior del grupo doméstico (Acuña, 2007).

Pese a la posición relativamente favorable que tienen las mujeres rarámuris en sus espacios domésticos -sobre todo si se les compara con las que guardan las mujeres pertenecientes a otros grupos étnicos-, su participación en el espacio público está sometida a restricciones de diversa índole. Las mujeres no tienen voz ni voto en las asambleas comunitarias y está vedado su acceso a los cargos de representación y autoridad. La diferenciación de funciones y actividades por género se aprecia de manera muy clara durante las celebraciones rituales (tesgüinadas y bailes), cuando se hacen evidentes las tareas y responsabilidades que son asignadas a cada uno de los sexos, así como sus respectivos espacios de interacción. Existen, sin embargo, referencias actuales de la participación de mujeres como representantes de las comunidades en cargos públicos creados por el Estado que requieren de diálogo e interacción con los mestizos o chabochis, o incluso como gobernadoras 
en los asentamientos establecidos en las sociedades de acogida (Martínez y Hernández, 2011). En cuanto a los cargos que poseen un fuerte valor simbólico, como los de owirúame (curandero) y wakiráame (cantador), éstos son ejercidos principalmente por hombres, aunque suele suceder que eventualmente alguna mujer pueda llegar a ocuparlos, siempre y cuando cuente con el reconocimiento social necesario.

Como se puede apreciar, contrario a lo que pudiera pensarse (Fagetti, 2002; Bonfil y Marcó del Pont, 1999), el sistema sexo-género rarámuri posee cierta flexibilidad en comparación con el de otros grupos indígenas (Gómez, 2009). La división del trabajo, por ejemplo, no se ajusta al patrón convencional que funciona en otros pueblos donde prevalece una economía de subsistencia basada en el agropastoreo. En el caso rarámuri, son las mujeres quienes generalmente ejercen de pastoras. En medio de la precariedad en que viven, las mujeres rarámuris mantienen cierto grado de independencia debido a que tradicionalmente se encuentran en igualdad de condiciones para heredar la tierra, poseer propiedades y tomar decisiones en el ámbito doméstico. ${ }^{2}$ Asimismo, en la esfera doméstica, son ellas quienes normalmente llevan la iniciativa en el matrimonio y en el divorcio o separación. Por otro lado, si bien sigue estando bajo su responsabilidad la carga productiva y reproductiva del hogar, en caso de necesidad los roles en el interior de la familia rarámuri pueden ser intercambiados.

Lo anterior ha llevado a algunos autores a postular la complementariedad de los roles y funciones de género en el plano práctico y simbólico, sustentada sobre la base de la "conciencia de dualidad", que forma parte de la cosmovisión rarámuri y que se expresa bajo dos maneras distintas de estar en el mundo: como hombre y como mujer (Acuña, 2007). Esta noción, permeada de un carácter marcadamente esencialista, niega la subordinación de género bajo el argumento de la "complementariedad de los opuestos" que está presente en la naturaleza, minimizando con ello el papel de las relaciones de poder, la inequidad y la desigualdad genérica entre las y los rarámuris.

No obstante, el régimen patriarcal de este grupo étnico queda en evidencia al analizar la forma en que está conformada la estructura de autoridad -y por tanto de poder- en las comunidades rarámuris, en donde los cargos de representación sólo pueden ser ocupados por hombres, y en donde existe

2 La noción de que existe una equidad absoluta en el reparto de la tierra y la herencia de otros bienes debe ser matizada. Investigaciones desarrolladas en contextos culturales similares, donde predominan sistemas testamentarios de tipo bilateral, han podido constatar la existencia de arreglos familiares que tienden a privilegiar la herencia hacia los hijos varones. Incluso algunos investigadores (Gómez, 2009) ubican a la propiedad de la tierra como parte de las proscripciones del mundo femenino rarámuri. 
toda una serie de tareas y actividades que a lo largo del ciclo de vida están restringidas para las mujeres, tales como la participación en las asambleas comunales, la participación en ciertos juegos y danzas tradicionales, y en general, en todas las ocupaciones que son propias del ámbito público y que poseen valor simbólico. De igual modo, las diferencias en el grado de escolaridad y de alfabetización entre hombres y mujeres que prevalecen en las comunidades no sólo denotan inequidad en el acceso a las oportunidades, sino que se convierten, además, en una de las principales limitantes para el desarrollo de las mujeres. A todo esto, habría que agregar la carga de la violencia doméstica, el machismo y el alcoholismo, que han reforzado el modelo patriarcal que predomina actualmente entre las y los integrantes de este grupo étnico.

\section{Reconfiguración de las identidades masculinas rarámuris}

Para los fines de este trabajo se entiende la masculinidad como el conjunto de atributos, valores, funciones y conductas que se suponen esenciales al hombre en una cultura determinada, y que es determinante en sus identidades, la definición de sus roles y asignaciones (Fernández, 2011; De Keijzer, 1997).

Si bien no es posible hablar de una masculinidad única -ya que éstas se construyen en el contexto de sistemas socioculturales concretos y con referencia a las adscripciones de clase, religión, etnia, identidad, entre otros-, es posible afirmar la existencia de una forma hegemónica de socialización cultural del ser masculino, que está cultural e históricamente construida, y que pese a tener sus variaciones, por clase o por etnia, se expresa en formas de organización, criterios de valoración y relaciones de poder asimétricas, de índole patriarcal, que se traducen en desigualdad genérica en el ámbito familiar, comunitario, económico, jurídico y social (Ortega, 2005).

La pertenencia étnica es uno de los principales referentes culturales que marcan diferencias significativas en la forma en que hombres y mujeres construyen sus identidades de género. No obstante, los conceptos y valores asociados a la masculinidad y la feminidad son históricos y se forman y transforman en el tiempo, a veces influidos por factores extragénero, como la modernidad, la tecnología, las crisis económicas y los procesos migratorios, entre otros, por lo que es necesario analizar las construcciones de género desde una perspectiva espacial y temporal amplia que permita dar cuenta de las dinámicas de cambio y permanencia.

En este sentido, cuando se habla de masculinidades rarámuris, se alude a las distintas formas en que los hombres de este grupo étnico integran de- 
finiciones, valores, creencias y significados acerca de lo que es y debe hacer un varón, así como de la posición que éstos detentan en relación con las mujeres.

Pérez (2003) refiere dos momentos históricos en donde la construcción masculina entre los rarámuris se ha visto modificada o resignificada. El primero tiene que ver con el proceso de evangelización, que en el caso de la etnia rarámuri se hizo presente a partir de la llegada de los jesuitas en el siglo XVII. La imposición de la religión católica, por parte de los conquistadores españoles, supuso la adopción de una estructura de gobierno cuyo ejercicio de poder es de tipo jerárquico, centralizado en torno al templo, y ejercido por una sola persona; todo ello en detrimento de las formas de organización tradicional de tipo comunitario (León, 1992). No es casual que la estructura de autoridad vigente entre los rarámuris hasta nuestros días, refleje en su terminología la permanencia de la influencia colonial y las formas de organización impuestas durante la dominación española: gobernador, general, capitán, mayora (de mayor), alguacil, entre otros. Tampoco es casual la proscripción que existe para que las mujeres ocupen estos cargos de autoridad en sus comunidades de origen. La adopción del catolicismo supuso también la incorporación de su currículum explícito e implícito, el cual planteaba la exclusión de las mujeres de la vida religiosa y pública en general, así como una concepción instrumental de la naturaleza, escindida del ser humano y puesta a su servicio.

La influencia misionera se manifestó también en la adopción de prejuicios corporales que estaban ausentes antes de la evangelización. Diversos investigadores señalan que el pudor que muestran actualmente las mujeres por mostrar el cuerpo -el vestido tradicional les cubre todo el cuerpo, incluida la cabeza-, no siempre fue tal. Carl Lumholtz (1994), por ejemplo, en las fotografías que tomó a finales del siglo XIX muestra a mujeres rarámuris con los pechos descubiertos. Actualmente, más del $90 \%$ de la población rarámuri está bautizada por el rito católico, lo que habla del grado de penetración de la influencia evangelizadora entre este grupo étnico.

El segundo momento histórico en el que la construcción social de la masculinidad rarámuri se vio transformada se vincula a la imposición de la figura jurídica del ejido por parte del Estado. Durante el reparto agrario de 1930 el territorio indígena fue dividido arbitrariamente en varios ejidos, sin respeto a los linderos naturales y ancestrales de las comunidades indígenas, perdiéndose con ello la propiedad legal de un solo territorio. Esta circunstancia derivó en la pérdida de poder y control de los pueblos indígenas sobre sus tierras, aun cuando quedaron integrados como parte del ejido. Esta nueva forma de tenencia de la tierra modificó la estructura de la propiedad 
comunal vigente en la Sierra Tarahumara e impuso una forma de organización social y productiva construida alrededor de un sujeto social concreto -el ejidatario varón-, el cual fue durante muchos años el único sujeto de derechos agrarios, ya que la ley mexicana hasta antes de 1971 no concedía a las mujeres el derecho de ser ejidatarias por derecho propio y poseer tierras, a menos que fueran viudas o recibieran la tierra por herencia (González, 2012). Esto significó que las mujeres no tuvieran históricamente ni voz ni voto en las asambleas ejidales y que quedaran excluidas de los procesos de toma de decisiones sociales y productivas en sus localidades. Aunque esta legislación fue posteriormente revertida, el pleno derecho a la titularidad de las parcelas ejidales sigue siendo una deuda pendiente.

La asamblea ejidal, así como los órganos de autoridad en el interior del ejido, se convirtieron en espacios de interacción masculina, así como de vinculación y contacto con la sociedad mestiza. Al paso del tiempo, los ejidatarios rarámuris asumieron muchas de las actitudes y valores promovidos por los mestizos que trabajaban en la Secretaría de la Reforma Agraria (SRA), la cual, con una clara orientación productivista, promovió durante décadas, por ejemplo, la remoción de masas forestales a partir del Programa Nacional de Ganaderización y la Comisión Nacional de Desmontes, entre los años sesenta y setenta, que provocó la desaparición del $80 \%$ de las selvas húmedas de México (González, 2012).

Las repercusiones de una política de reparto agrario que no respetó la visión y cultura rarámuris han sido devastadoras en términos sociales y ambientales. Los conflictos comunitarios se multiplicaron como resultado de la formación de grupos de poder que se fortalecieron al amparo de los órganos de autoridad del ejido; se erosionaron algunas prácticas comunitarias, como la toma de decisiones por consenso, la cual fue sustituida por la votación mayoritaria en el seno de la asamblea ejidal; y se promovió una gestión forestal que favoreció a las minorías no indígenas, marginando a los rarámuris y a otros grupos étnicos de la sierra, lo que también ocasionó degradación forestal, pobreza e innumerables conflictos internos. Para tener una idea del predominio del ejido, basta con señalar que la Sierra Tarahumara tiene una superficie forestal total de 7587127 ha, de las cuales 5994308 ha son de tipo ejidal y pertenecen a 360 ejidos (González, 2012).

A partir de la revisión de la literatura sobre los procesos migratorios en el noroeste de México, así como del trabajo de campo desarrollado durante 2010 y 2011 en los campos agrícolas de Cuauhtémoc, Chihuahua, fue posible identificar un tercer momento histórico en que las masculinidades rarámuris se vieron nuevamente resignificadas. Dicho momento tuvo como marco el proceso de modernización agrícola acaecido en las regiones aleda- 
ñas a la Sierra Tarahumara, el cual inició durante la segunda mitad del siglo XX y se profundizó durante las décadas de los ochenta y noventa con la apertura comercial y la inserción de amplias regiones agrícolas del norte del país en los mercados globalizados. Los cambios en las relaciones de género y en las construcciones sociales vinculadas a la masculinidad, que resultaron de la incorporación de la etnia rarámuri a los mercados de trabajo desregulados y flexibles propios del modelo agroindustrial, se analizan en el siguiente apartado.

\section{Cambios en la identidad y las relaciones de género a partir de la experiencia migratoria}

El contexto de pobreza, deterioro ambiental y abandono institucional de las comunidades ubicadas en la Sierra Tarahumara propició, a mediados del siglo pasado, la paulatina integración de la población rarámuri a los circuitos migratorios de tipo pendular que tienen por destino las zonas urbanas y los campos agrícolas de la región, en los que se contratan como jornaleros durante los periodos de cosecha de fruta y verdura.

A partir de este proceso, las familias rarámuris se volvieron dependientes del trabajo asalariado y de las relaciones con la población mestiza. Al mismo tiempo se intensificaron la descomposición social, la adopción de patrones de consumo acordes con el modo de vida urbano y el menoscabo de los referentes identitarios tradicionales.

Si bien las vivencias que hombres y mujeres experimentan durante el tránsito y la estancia migratoria tienen elementos en común, su impacto y la manera en que la experiencia migratoria es vivenciada están claramente diferenciadas por género.

En efecto, la movilidad geográfica supone el intercambio no sólo de tecnologías, bienes materiales y mercancías, sino también de ideas, normas, símbolos y diversas expresiones del espacio donde se nace y se vive; de esta manera, la migración repercute en múltiples aspectos de la vida social y cotidiana, tales como: la estructura familiar, las relaciones interpersonales, los roles de género, las actividades productivas, los modos de vida y las identidades (Rea, 2007).

Entre los principales rasgos de la identidad que se refuerzan a partir de la integración a los circuitos migratorios se encuentran los mandatos de género, que en el caso de los hombres establecen la obligación de proveer económicamente al hogar, responsabilidad que en las localidades de origen es compartida por ambos cónyuges. Para muchos varones, el ingreso que 
generan como jornaleros les permite acatar los mandatos de género vinculados a su rol como proveedores, mismo que en el contexto de explotación, pobreza y vulnerabilidad de la Sierra Tarahumara resulta difícil de cumplir.

Cada año vengo aquí a trabajar, desde hace como unos diez años vengo con otros cinco compañeros. Cuando se acaba la manzana, vamos a Sinaloa, ahí [se cultiva] el jitomate, los chiles, todo. A mi comunidad voy cada tres semanas, paso tres meses en Sinaloa, luego en la casa estoy un mes para sembrar, tengo siembra, nomás puro maíz, frijoles, papas y luego [vengo] para acá. Tengo cinco hijos, para mantenerlos es muy difícil, ahí ando batallando, trabajando para que estudien, porque los que no están estudiados se ponen más borrachos. El que se pone a estudiar no se pone borracho porque sabe. Entonces pues de lo [que sale] de aquí compramos pantalones y zapatos, cuadernos [...] [Miguel, 37 años, originario de Bocoina, Chih.].

Por otro lado, en el trabajo en los campos agrícolas se fortalecen y reproducen también estereotipos y roles de género. En las huertas de manzana, por ejemplo, las mujeres se encuentran al margen de las actividades que demandan una mayor fortaleza física; tal es el caso de las labores de la temporada de pizca, una de las más largas y mejor retribuidas del proceso productivo, en donde existe una marcada preferencia por la contratación de varones, sobre todo durante las primeras semanas, cuando la cosecha no es voluminosa. Además, la carencia de servicios de guardería y las restricciones a la contratación de mujeres en ciertas épocas del año, propician que en muchas ocasiones ellas deban quedarse al cuidado de los hijos y garantizar la alimentación de las y los integrantes del grupo doméstico, mientras el esposo se encuentra en los campos agrícolas. Incluso en los casos donde las mujeres viajan con su grupo doméstico extenso con el objetivo de vender su fuerza de trabajo como jornaleras, el cuidado de niños y niñas queda a cargo de otras mujeres parientes o vecinas de su comunidad. Se aprecia en este caso cómo el mercado de trabajo agroindustrial, configurado a partir del género, contribuye a la reproducción de las desigualdades en el interior del hogar, al tiempo que precariza las condiciones bajo las cuales se accede al empleo.

Alejados de la dinámica social que prevalece en sus localidades de origen, y enfrentados a condiciones de vida y de trabajo extremas, algunos indígenas migrantes varones recurren al consumo cotidiano de bebidas alcohólicas. El pago por jornal les permite contar con recursos que gastan en la adquisición de bebidas alcohólicas adulteradas que nada tienen que ver con el tesgüino, bebida tradicional elaborada a base de maíz que se consume 
de manera ritual en la Sierra Tarahumara. Dichas bebidas, denominadas por los indígenas como "alcohol malo" o "gasolina", suelen causar estragos en la salud física y mental de los rarámuris, sin contar los perjuicios económicos inherentes a la adicción. Además del alcohol, se presenta, sobre todo entre la población más joven, el consumo de drogas como la marihuana, los solventes químicos y otras sustancias adictivas, con las que la población migrante entra en contacto a su llegada a la ciudad. En este sentido, se puede afirmar que en los espacios migratorios emergen prácticas inéditas que derivan del establecimiento de nuevas relaciones sociales y la reconstrucción de las identidades que tiene lugar a partir de la interseccionalidad con elementos de desigualdad, como el género, la etnia y la clase. Asimismo, se reproduce la denominada "masculinidad como factor de riesgo", entendida como la constitución misma de la masculinidad y de los contenidos identitarios de los hombres en las culturas indígenas y mestizas que los hacen más proclives a contraer ciertos tipos de enfermedades o a tener comportamientos de alto riesgo, que se traducen en accidentes, enfermedades o muerte, como consecuencia de la violencia, el alcoholismo y la drogadicción (Núñez, 2011).

Como se puede apreciar, la integración de elementos de la cultura dominante en el espacio migratorio ha implicado, entre otras cosas, la asimilación de los roles genéricos femeninos y masculinos tradicionales del sistema sexo/género mestizo. A su vez, ha propiciado el reforzamiento de los elementos patriarcales contenidos en su propia cultura, así como de estereotipos y valores masculinos, tales como: ser el más fuerte, el más inteligente, el que provee, el que trabaja, el que sí vale, el que decide, el que tiene libertad para salir, el que puede hablar en voz alta, así como golpear y defender su propiedad, incluidas su mujer y familia (Pérez, 2003).

Otro de los aspectos que se observan como una constante, tanto en las localidades de origen como en el espacio migratorio, es la normalización de la violencia dirigida hacia las mujeres. Dicha violencia tiene su origen en la desigualdad que existe entre hombres y mujeres, y en la discriminación que existe hacia ellas, constituyendo un recurso más de la dominación. Los hombres afirman su superioridad demostrando fuerza y control hacia las mujeres para dirigir, dominar y gobernar a través de un proceso de socialización que tiene como objetivo cumplir con los mandatos que se asocian a la virilidad (Martínez y Mejía, 1997). La población de origen rarámuri no escapa a este tipo de relaciones de dominación/subordinación cuya expresión más radical es la violencia física, a la que se suma la económica, que forma parte de la denominada violencia estructural de género. 
Aquí nos han dicho que las mukiras [muchachas] no salgan mucho para afuera [del albergue] porque es peligroso; ahí otra persona puede que las agarren allá afuera, las matan o violan; los abusadores pueden ser tarahumaras, chabochis, de todo. En el albergue nos quedamos mejor [Nazaria, 16 años, originaria de Carichi, Chih.].

Los testimonios de violencia de género en el interior del grupo doméstico entre la población rarámuri son ilustrativos de la forma en que este tipo de conductas son reproducidas y de algún modo naturalizadas por hombres y mujeres rarámuris desde la edad más temprana. La gran cantidad de referencias con respecto a este tema es un indicativo de la presencia generalizada de una cultura de violencia hacia las mujeres, la cual está sumamente extendida entre la población rarámuri.

A las mujeres no las tratan bien, a veces las golpean, a mí también [me han golpeado] nomás por celos; dice que yo anduve con tal hombre, aunque no sea cierto. Siento feo, no me escucha, a nadie le digo porque no puedo, me da pena, sólo me aguanto, será porque casi no está conmigo en el pueblo, se va para Sinaloa a trabajar, no sé cuándo regrese. Ahora tengo tres meses que no lo veo, por eso vine a trabajar, para comer algo [Alicia, 26 años, originaria de Lagunita, Uruachi, Chih.].

En la Sierra Tarahumara las autoridades tradicionales, conformadas en su mayoría por varones, suelen minimizar la violencia de género mediante la imposición de sanciones que no corresponden a la gravedad de la agresión. De esta forma, aunque en la cosmovisión rarámuri las mujeres ocupan una posición de privilegio, respeto y complementariedad, los usos y costumbres no castigan la violencia que sus familiares o parejas ejercen en su contra.

En los espacios migratorios la incidencia o gravedad de la violencia de género también está presente, aunque con diferentes matices. A veces se observa de forma explícita, y otras tantas, enmascarada bajo formas de agresión, subordinación o dominio que, al estar integradas a un sistema genérico patriarcal, resultan menos evidentes. Tal es el caso del aislamiento social, la reclusión en el espacio doméstico, las restricciones a la movilidad y la exclusión de la toma de decisiones con respecto al destino de los ingresos familiares.

Si bien es cierto que la vulnerabilidad de las mujeres puede incrementarse durante la estancia migratoria por la ausencia de lazos familiares y comunitarios que les brinden soporte, algunas encuentran apoyo en las instancias de mediación y amparo presentes en los entornos urbanos, las cuales les permiten afrontar las agresiones y abusos por parte de sus parientes y/o 
cónyuges. La asistencia que las instituciones gubernamentales y civiles otorgan a las mujeres víctimas de violencia contrasta con la complicidad de género presente en sus localidades de origen, donde las agresiones hacia las mujeres suelen ser toleradas e invisibilizadas por las autoridades tradicionales. Lo anterior supone cambios en la conducta de hombres y mujeres con respecto a la agresividad y la violencia, al menos en los espacios migratorios.

Aquí en el albergue hay mucha familia, niños, mujeres; si viene alguno que quiere entrar aquí borracho a molestar o pegar, pues se le habla a la policía y se lo llevan. Aquí no debe uno de tomar, este albergue no es para tomar, es para vivir, para la gente que viene a trabajar, porque en un albergue hay mucha familia; si pasara algo aquí, no, no [pasa] gracias a que no toman [Pedro, 52 años, Albergue Minita, originario de Baqueachi, Carichí, Chih.].

Por otro lado, la inserción de las mujeres en el mercado laboral obliga a replantear la organización y distribución del trabajo reproductivo en los hogares, lo que puede dar lugar a cambios en las relaciones de dominio y subordinación entre los géneros, $y$, por tanto, a la reconfiguración de las identidades de género, entre ellas, las referentes a la masculinidad.

Tengo como 15 años viniendo y regresando; hace poco fui a la sierra, me quedé como tres semanas cultivando frijolito y maicito. Aquí [en la ciudad] las mujeres lavan, también a veces los hombres ayudan a lavar, también a lavar o a cocinar también. Cuando mi mujer no tiene chance, a veces cocino; cuando llego con hambre, pues a cocinar y a cenar [Pedro, 52 años, Albergue Minita, procedente de Baqueachi, Carichí, Chih.].

Algunas mujeres refieren que la migración, más allá de los costos sociales y humanos que conlleva, les ha permitido ampliar sus espacios de acción y mejorar sus capacidades de negociación, tanto en sus comunidades como dentro de sus grupos domésticos. Muchas de ellas afirman que se comunican mejor con sus parejas y que toman más decisiones en forma conjunta que cuando vivían en sus localidades de origen. Todo ello habla de cambios en las relaciones de género y, por tanto, en las construcciones de lo masculino y lo femenino.

Antes sí era muy diferente, antes el que mandaba era el hombre nada más, el hombre era el que decía, el que decidía qué se tiene que hacer. Últimamente ha ido cambiando, ya la mujer ya también puede pues actuar, ya no se deja tan fácilmente de que el hombre es el que manda. Yo digo que ha cambiado, ha habido ese respeto [Samuel, 30 años, rarámuri asentado]. 
En este sentido, se puede afirmar que las nuevas pautas de socialización que impone la experiencia migratoria tienen el potencial de replantear las nociones tradicionales de género y favorecer el tránsito hacia modelos menos inequitativos y autoritarios.

Existen también elementos para afirmar que en el contexto migratorio la normatividad conyugal entre la población de origen rarámuri se ha flexibilizado. Una de las principales consecuencias de las prácticas migratorias es que multiplican las oportunidades de conocer personas de otras localidades. En los campos agrícolas es frecuente encontrar hombres y mujeres rarámuris que se separaron de su primera unión y que en la ruta migratoria encontraron una nueva pareja con la cual se establecen fuera de sus localidades de origen. En muchas ocasiones esta pareja es mestiza, o pertenece a otro grupo étnico, lo que implica la asimilación de distintos valores, normas, formas de comportamiento, símbolos y significados asociados con las relaciones de género.

Otro factor que ha generado cambios en las identidades de género, al tiempo que ha contribuido a mitigar los efectos de la violencia de género entre la población rarámuri, es la migración definitiva y el consiguiente cambio de residencia. Actualmente existe un número creciente de jóvenes rarámuris que, al iniciar su vida conyugal, deciden establecerse en las zonas urbanas adyacentes a los campos agrícolas, en compañía de otros parientes o parejas que también han migrado. Los ingresos provenientes de la venta de su fuerza de trabajo les ofrecen la posibilidad de vivir de manera independiente, rompiendo con el patrón de residencia patrivirilocal, que en sus comunidades de origen suele ser fuente de presiones y conflictos, sobre todo para las mujeres jóvenes.

Otro aspecto interesante que se presenta en los asentamientos rarámuris urbanos es el abandono por parte de los hombres de los espacios tradicionales de autoridad. En este tipo de asentamientos es frecuente que el cargo de "gobernador" sea ocupado por mujeres. En el contexto de las sociedades de acogida, este cargo supone principalmente el desarrollo de actividades asociadas con el cuidado: salud, educación, alimentación y saneamiento ambiental. Las "gobernadoras indígenas", en su calidad de representantes de la población rarámuri, suelen llevar a cabo ante las autoridades estatales y municipales los trámites y gestiones necesarias para la dotación de infraestructura y servicios públicos en los albergues y asentamientos donde viven. La población migrante de origen rarámuri recurre a ellas para solucionar problemas internos o externos, para que hablen a su nombre frente a las autoridades y funcionarios, para gestionar diversos tipos de apoyo, o para que sirvan como traductores o interlocutores frente a los mestizos. Esta tarea 
supone un despliegue considerable de tiempo y esfuerzo, ya que adicionalmente deben cumplir con las actividades de carácter reproductivo en sus respectivos hogares y con su labor como jornaleras en los campos agrícolas. Lo anterior deriva en una carga extraordinaria de trabajo (triple jornada) que no suele ser objeto de remuneración, situación que las coloca en una posición económica vulnerable, dado que, independientemente del cargo que ostentan, su situación de pobreza es similar a la de sus representados (Martínez y Hernández, 2011).

Por otro lado, muchos de los programas y organismos sociales que atienden a la población rarámuri en las ciudades canalizan sus recursos y apoyos institucionales a través de la participación de estas mujeres, sin considerar que la gestión de los mismos representa una responsabilidad adicional que deriva en un incremento de las cargas de trabajo, y que la asignación de tareas vinculadas a la reproducción (servicio, atención, cuidado, etc.) replica, a nivel institucional, una visión estereotipada acerca de los roles que deben cumplir hombres y mujeres en el sistema tradicional de género (Nava, 2007).

El hecho de que los hombres cedan este tipo de espacios de autoridad a las mujeres no es una concesión. Desempeñar el cargo de "gobernador" en un contexto urbano, implica erogar una gran cantidad de recursos económicos y de tiempo, por lo que para muchos varones esta actividad ha perdido valor. No obstante, para las mujeres rarámuris asumir el cargo de "gobernadora" constituye una oportunidad para obtener posiciones de representación social y prestigio que en sus localidades de origen les han sido tradicionalmente negadas. Al mismo tiempo, representa un pretexto para la movilidad, así como para el desarrollo y aprendizaje de nuevas habilidades.

Como gobernadora de la colonia si veo que [los esposos] golpean a la mujer, los mando a la cárcel; ya aquí ya casi no [lo hacen], ya se controlaron. Si, por ejemplo, a media noche, ¿qué pasó?, ¿se están peleando? pues mejor pido la patrulla, que duerman allá, y al otro día que se arreglen. Si golpean a las mujeres, trato de platicar con ellos, hacemos reuniones, [les digo] que no deben de ser así; también me ocupo de acompañar a gente que viene de la sierra a los hospitales, porque no saben decir qué les duele, o a meter solicitudes para ayudar a la gente [Josefina, 38 años, asentamiento rarámuri, Cuauhtémoc, Chih.].

Como se puede apreciar, la creciente participación de las mujeres en el ámbito productivo -y por lo tanto, remunerado-, así como en los espacios de toma de decisión a nivel comunitario dentro de las sociedades de acogida, ha implicado transformaciones importantes en la dinámica del grupo doméstico y en la reconstitución de las identidades de género. Frente a este fenó- 
meno, las masculinidades no permanecen estáticas y asumen diversas vías, algunas de las cuales pueden derivar en el tránsito hacia modelos más equitativos y menos autoritarios.

En este sentido, es posible señalar que la migración abriga la potencialidad de alterar algunas de las asimetrías tradicionales entre hombres y mujeres pertenecientes al grupo étnico rarámuri; aunque es necesario reconocer que los cambios que induce no necesariamente son siempre positivos, y que éstos dependen de una serie de factores conexos contingentes a la situación migratoria de cada grupo doméstico.

\section{Conclusiones}

A pesar de que algunos investigadores (Acuña, 2007; Gómez, 2009) plantean que el sistema sexo/género rarámuri posee cierto grado de flexibilidad con respecto a la división del trabajo y las relaciones afectivo sexuales, las mujeres rarámuris se encuentran inmersas en un modelo sociocultural que se caracteriza por reproducir el dominio masculino. Este modelo patriarcal descansa en varias proscripciones que sufre el mundo femenino rarámuri: a nivel familiar la toma de decisiones compete únicamente a los varones; las mujeres no pueden participar en las asambleas ni asumir ningún cargo político, y les están vedadas las posiciones y actividades dotadas con valor simbólico. El predominio masculino de esta sociedad es visible en la competencia sanadora de los curanderos, en las danzas varoniles de matachines, pintos y fariseos, y en la mayoría de los juegos tradicionales que están reservados sólo para los hombres; también en la incidencia de la violencia de género, fenómeno sumamente extendido tanto en la Sierra Tarahumara como en el espacio migratorio.

A lo largo de la historia, la construcción y reconstrucción de las masculinidades rarámuris se han visto modificadas o resignificadas por la influencia de tres instancias sociales: la Iglesia con el proceso de evangelización que tuvo lugar en el siglo XVII; el Estado con el reparto agrario y la imposición de la figura jurídica del ejido durante el siglo XX; y el mercado agrícola, cuya dinámica actual se asocia a la producción intensiva de cultivos comerciales que demandan fuerza de trabajo de tipo estacionaria, con pocos trabajadores permanentes.

La reconfiguración contemporánea de las masculinidades rarámuris ocurre en el marco del proceso de modernización agrícola y la profundización del modelo económico neoliberal que, sumado al proceso de empobrecimiento asociado a la sobreexplotación forestal, a los cambios ambientales y a la 
profundización de la violencia estructural, entre otros aspectos, han favorecido la integración de la población rarámuri a los circuitos migratorios, propiciando con ello cambios en valores, normas, formas de comportamiento, símbolos y significados asociados con las relaciones de género.

En efecto, la migración constituye hoy en día una de las estrategias de reproducción más importantes entre los grupos domésticos rarámuris, la cual les ha permitido sobrevivir al hambre endémica que se presenta en muchas comunidades de la Sierra Tarahumara. Este proceso ha dado pie a reconfiguraciones en las masculinidades rarámuris, las cuales, lejos de mostrarse estáticas, se han reconstituido a partir del contacto con otros contextos culturales y de los cambios de orden socioeconómico que conllevan los procesos migratorios de tipo estacional o permanente.

A partir del trabajo realizado con mujeres y hombres, jornaleros agrícolas migrantes, se puede afirmar que, aunque las masculinidades rarámuris comparten algunos elementos del modelo hegemónico patriarcal -como las prácticas y las significaciones que establecen las distancias jerárquicas y las relaciones de dominación de los hombres respecto a las mujeres-, la construcción social del género masculino en el interior de esta etnia no es homogénea. Los cambios inducidos por la migración han dado lugar a la conformación de diversas configuraciones de las masculinidades, las cuales obedecen a contextos socioeconómicos, familiares y culturales particulares, en los que las construcciones de lo masculino y lo femenino generan diversas prácticas y formas de relacionarse desde el género.

La asimilación de valores, normas, significados y pautas de comportamiento inéditas, asociadas con las relaciones de género, se aprecian en aspectos tales como: la incorporación de las mujeres al trabajo remunerado, la reorganización y distribución del trabajo doméstico en los hogares, la ampliación de la participación femenina en los espacios de toma de decisiones comunitarios y la contención de la violencia de género, asociada en buena medida a las instituciones que atienden a las mujeres víctimas de violencia en las sociedades de acogida.

En este sentido, vale la pena señalar que, si bien la experiencia migratoria tiene el potencial de replantear las nociones tradicionales de género y favorecer el tránsito hacia modelos menos inequitativos y autoritarios, los cambios identitarios que de ella se derivan ocurren en el marco de un modelo hegemónico en el que se presenta al varón como esencialmente dominante, con componentes que emanan de la ideología, los valores y los principios rectores del patriarcado. 


\section{Bibliografía}

Acuña, A. (2007). La mujer en la cosmovisión y ritualidad rarámuri. Boletín de Antropología, 21(38), 41-63. Recuperado de http://www.redalyc.org/ pdf $/ 557 / 55703803 . p d f$

Acuña, Á. y Gómez, E. (2012). Papeles de género en la sexualidad rarámuri: una aproximación etnológica. Revista de Antropología Experimental, 12, 1-15. Recuperado de http://revista.ujaen.es/huesped/rae/articulos 2012/01acuna12.pdf

Bennett, W. y Zingg. R. M. (1978). Los tarahumaras, una tribu india del norte de México. Ciudad de México: Instituto Nacional Indigenista.

Bonfil, P. y Marcó del Pont, R. (1999). Las mujeres indígenas al final del milenio. Ciudad de México: FNUAP / Conmujer.

Cárdenas, E. (2014). Migración interna e indígena en México: enfoques y perspectivas. Intersticios Sociales, 7, 1-28. Recuperado de http://www. scielo.org. $\mathrm{mx} / \mathrm{scielo} . \mathrm{php}$ ?script $=$ sci_arttext\&pid=S2007-4964 2014000100003

Connell, R. W. y Messerschimdt, J. W. (2005). Hegemonic masculinity. Rethinking the concept. Gender \& Society, 19(6), 829-859. Recuperado de http://journals.sagepub.com/doi/pdf/10.1177/0891243205278639

De Keijzer, B. (1997). El varón como factor de riesgo: masculinidad, salud mental y salud reproductiva. En E. Tuñón (coord.), Género y salud en el sureste de México. Villahermosa, Tabasco, México: Ecosur / UJAT.

Díaz, R. (2011). Migración, etnia y género en la sobrevivencia y (re)significación masculina Xi'oi-pame (Tesis de doctorado en Estudios Internacionales e Interculturales, Universidad del Deusto, España).

Díaz, R. (2014). La perspectiva de género en la comprensión de la masculinidad y la sobrevivencia indígena en México. Agricultura, Sociedad y Desarrollo, 11(3), 359-378. Recuperado de http://www.scielo.org.mx/ scielo.php?script $=$ sci_arttext\&pid=S1870-54722014000300006

Fagetti, A. (2002). Pureza sexual y patrilocalidad: el modelo tradicional de familia en un pueblo campesino. Alteridades, 12(24), 33-40. Recuperado de http://www.redalyc.org/html/747/74702403/

Fernández, A. (2011). Prejuicios y estereotipos. Antropología Experimental, 11(22), 317-328. Recuperado de https://revistaselectronicas.ujaen.es/ index.php/rae/article/view/1932/1682

Gabrielova, Z. (2007). Los rarámuri: un pueblo indígena de México (Tesis de licenciatura. Universidad de Masaryk, Facultad de Artes, Brno, República Checa). Recuperado de http://is.muni.cz/th/137848/ff_b/Los_ raramuriun_pueblo_indigena_de_Mexico.pdf 
Gobierno del Estado de Chihuahua. (2006). Pueblos indígenas de la Sierra Tarahumara. Gobierno del Estado de Chihuahua. Disponible en http:// www.chihuahua.gob.mx (13 de julio de 2017).

Gómez, A. (2009). El sistema sexo / género y la etnicidad: sexualidades digitales y analógicas. Revista Mexicana de Sociología, 71(4). Recuperado de http://www.scielo.org.mx/scielo.php?script=sci_arttext\&pid $=\mathrm{S} 0188-25032009000400003$

González, B. (2012). La Sierra Tarahumara, el bosque y los pueblos originarios: estudio de caso de Chihuahua (Documento de trabajo). Roma, Italia: Food and Agriculture Organization (FAO). Recuperado de http:// www.fao.org/forestry/17194-0381f923a6bc236aa91 ecf614d92e12e0. pdf (26 de abril de 2016).

INALI (2010). Indicadores básicos de la agrupación tarahumara, 2010. Ciudad de México: Instituto Nacional de Lenguas Indígenas. Recuperado de https://site.inali.gob.mx/Micrositios/estadistica_basica/estadis ticas2010/pdf/agrupaciones/tarahumara.pdf (20 de marzo de 2018).

INEGI (2010). Censo de Población y Vivienda 2010. Aguascalientes, México: Instituto Nacional de Estadística y Geografía. Recuperado de http:// www.beta.inegi.org.mx/programas/ccpv/2010/

Kennedy, J. (1970). Inápuchi. Una comunidad tarahumara gentil. Ciudad de México: Instituto Indigenista Interamericano.

Lara, S. (1998). Nuevas experiencias productivas y nuevas formas de organización flexible del trabajo en la agricultura mexicana. Ciudad de México: Juan Pablos Editores / Procuraduría Agraria.

Lara S. y Carton de Grammont, H. (2011). Reestructuraciones productivas y encadenamientos migratorios en las hortalizas sinaloenses. En S. Lara (coord.), Los encadenamientos migratorios en espacios de agricultura intensiva. Ciudad de México: Miguel Ángel Porrúa.

León, R. (1992). Misiones jesuitas en la Tarahumara (siglo XVIII). Ciudad Juárez, México: Universidad Autónoma de Ciudad Juárez.

Lumholtz, C. (1994). El México desconocido. Chihuahua, México: Ayuntamiento de Chihuahua.

Martínez, B. y Hernández, A. (2011). El reto de la interculturalidad y la equidad de género en la migración jornalera rarámuri. Ciudad de México: Indesol / Colegio de Postgraduados.

Martínez, B. y Mejía, S. (1997). Ideología y práctica en delitos cometidos contra mujeres. El sistema judicial y la violencia en una región indígena. Puebla, México: Colegio de Postgraduados.

Nava, M. (2007). Relaciones de género: población jornalera agrícola migrante. En INM (coord.), Mujeres afectadas por el fenómeno migratorio en 
México. Una aproximación desde la perspectiva de género (Memoria). Ciudad de México: Instituto Nacional de las Mujeres.

Neumann, J. y González, L. (1991). Historia de las rebeliones de la Sierra Tarahumara (1626-1724). México: Camino.

Núñez, G. (2011). Hombres indígenas, diversidad sexual y vulnerabilidad al VIH-sida: una exploración sobre las dificultades académicas para estudiar un tema emergente en la antropología. Desacatos, 35, 13-28. Recuperado de http://desacatos.ciesas.edu.mx/index.php/Desacatos/article/ view/318/198

Ortega, M. (2005). Masculinidad y factores socioculturales asociados al comportamiento de los hombres frente a la paternidad en Centroamérica. Santiago de Chile: CEPAL.

Pérez, J. C. (2003). La variable de la masculinidad en los procesos para el desarrollo sustentable, experiencia y marco teórico. La Ventana. Revista de Estudios de Género 17, 250-302. Recuperado de http://148.202.18.157/ sitios/publicacionesite/pperiod/laventan/Ventana17/17-8.pdf

Pintado, A. P. (2000). Tarahumaras, pueblos indígenas del México contemporáneo. Ciudad de México: Comisión Nacional para el Desarrollo de los Pueblos Indígenas.

PNUD (2010). Informe sobre Desarrollo Humano de los Pueblos Indígenas en México. Ciudad de México: Programa de las Naciones Unidas para el Desarrollo.

Raesfeld, L. (2006). Características culturales de los grupos de jornaleros migrantes. En Situación socioeconómica, cultural y educativa de niños que pertenecen a familias de jornaleros agrícolas en el estado de Hidalgo (Informe). Pachuca, Hidalgo, México: Universidad Autónoma del Estado de Hidalgo.

Rea, P. (2007). Reflexiones en torno a la migración femenina y su impacto en las relaciones de género. En INM (coord.), Mujeres afectadas por el fenómeno migratorio en México. Una aproximación desde la perspectiva de género (Memoria). Ciudad de México: Instituto Nacional de las Mujeres.

Rodríguez, J. (1999). Las carreras rarámuri y su contexto: una propuesta de interpretación. Alteridades, 9(17), 127-146. Recuperado de http://biblio teca.ues.edu.sv/revistas/10800285-12.pdf

Rubin, G. (1986). El tráfico de mujeres: notas sobre la economía política del sexo. Nueva Antropología, 8(30), 95-145. Recuperado de http://www. caladona.org/grups/uploads/2007/05/El\%20trafico\%20de\%20mujeres2. pdf

Stefani, P. y Arteaga, A. (1995). Geografía y población de la Sierra Tarahu- 
mara. En L. González et al. (coords.), Derechos culturales y derechos indigenas en la Sierra Tarahumara. Ciudad Juárez, Chih.: Universidad Autónoma de Ciudad Juárez.

Velasco, P. (1987). Danzar o morir: religión y resistencia a la dominación en la cultura tarahumara. Ciudad de México: Centro de Reflexión Teológica

\section{Acerca de los autores}

Beatriz Martínez Corona es doctora en Ciencias con especialidad en Estrategias de Desarrollo Agrícola Regional y maestra en Ciencias en Estudios del Desarrollo Rural, ambos grados por el Colegio de Postgraduados; es licenciada en Psicología por la Universidad Nacional Autónoma de México. Ha realizado estancias de investigación y actualización en la Universidad de Durham (Inglaterra) y en el Departamento de Antropología Cultural de la Universidad de Utrecht (Holanda), así como una estancia sabática en la Universidad Politécnica de Madrid. Es integrante del Sistema Nacional de Investigadores, nivel II. Es profesora investigadora del Colegio de Postgraduados, y docente en el programa Estrategias de Desarrollo Agrícola Regional y en la Maestría en Gestión del Desarrollo Social. Desarrolla proyectos de investigación en torno a género, ambiente, transformaciones sociales y participación de mujeres y hombres en procesos de desarrollo, análisis de políticas públicas, educación desde la perspectiva de género, e interculturalidad. Ha coordinado diversos proyectos de investigación, y ha dirigido y asesorado múltiples trabajos de tesis de doctorado y maestría. Ha publicado múltiples artículos y libros; también ha colaborado en la inclusión de la perspectiva de género en las políticas públicas. Participa en asociaciones académicas nacionales e internacionales. ORCID: http://orcid.org/00000002-0745-4270

José Álvaro Hernández Flores es doctor en Ciencias con especialidad en Estrategias para el Desarrollo Agrícola Regional por el Colegio de Postgraduados y licenciado en Economía por la Universidad Nacional Autónoma de México. Es miembro del Sistema Nacional de Investigadores, nivel I. Ha desarrollado estancias de investigación en el Instituto Gino Germani de la Universidad de Buenos Aires y en el Centro de Estudios del Desarrollo Económico y Social (CEDES) de la Benemérita Universidad Autónoma de Puebla. Actualmente se encuentra adscrito como catedrático Conacyt al Centro de Estudios Demográficos, Urbanos y Ambientales de El Colegio de 
México, A.C. Entre sus principales líneas de investigación destacan: desarrollo sustentable, estrategias de reproducción social, y configuración de los territorios rurales y periurbanos. ORCID: https://orcid.org/0000-0002-83490505

Recepción: 17 de julio de 2017.

Aceptación: 20 de abril de 2018. 
